\title{
GENERALIZED CAUSE AND EFFECT DEMONSTRATION PROTOCOL
}

\author{
ROBERT W. BASS \\ Innoventech Inc., P.O. Box 1238, Pahrump, NV 89041-1238 \\ E-mail: donquixote@innoventek.com
}

MICHAEL C. H. MCKUBRE

SRI International, Menlo Park, CA

E-mail: michael.mckubre@sri.com

\begin{abstract}
It is universally accepted, even by nonscientists, that if the measured output from a physical system is double, triple, or quadruple that obtained when the measured stimulus/input is doubled, tripled, or quadrupled then there is a "cause and effect relationship" between the input and output (e.g. total energy input versus excess energy [or nuclear ash] output in a cold fusion experiment). How does one quantify this scientifically, when random process disturbances and random measurement noises preclude perfect linearity? This question is answered under the assumption of Gaussian (or "normal") departures from ideal linearity regarding the two mentioned statistical aspects. This is a generalization of the protocol proposed by Bass [1], which is more realistically flexible in several respects. An arbitrary number $N \geq 3$ of similarly prepared samples is allowed, and neither the voltage nor the current is required to be constant. However, the previous protocol may be recovered as a special case when $N=5$.
\end{abstract}

\section{Introduction}

The following protocol applies to the repetitive conduct (allowing for a control blank and at least 3 actual non-trivial input/output experiments) of an experiment, each example of which is characterized by just two numbers, called an input cause $C$ and an output effect $E$. Each pair $(C, E)$ will be called a "sample." For utmost mathematical generality we shall consider as few as 3 samples, but in the usual case where $(C, E)=(0,0)$ in the case of a control blank, there would then be only two meaningful samples and their plotting on an approximately straight line through the origin in the $(C, E)$ plane will not be as convincing as when many samples are plotted, because in the presence of stochastic disturbances and noise a single appearance of near-linearity could be ascribed to mere "coincidence." The purpose of the present protocol is to enable a statistically meaningful Confidence Limit to be assigned rigorously to the "goodness of fit to an ideal straight line," as depicted in the hypothetical example of Fig. 1, based upon a computer simulation using pseudo-random stochastic processes in the case of $N=6$ samples. It was originally proposed by Bass [1] to take $N=5$ and to modulate the inputs to be integral 


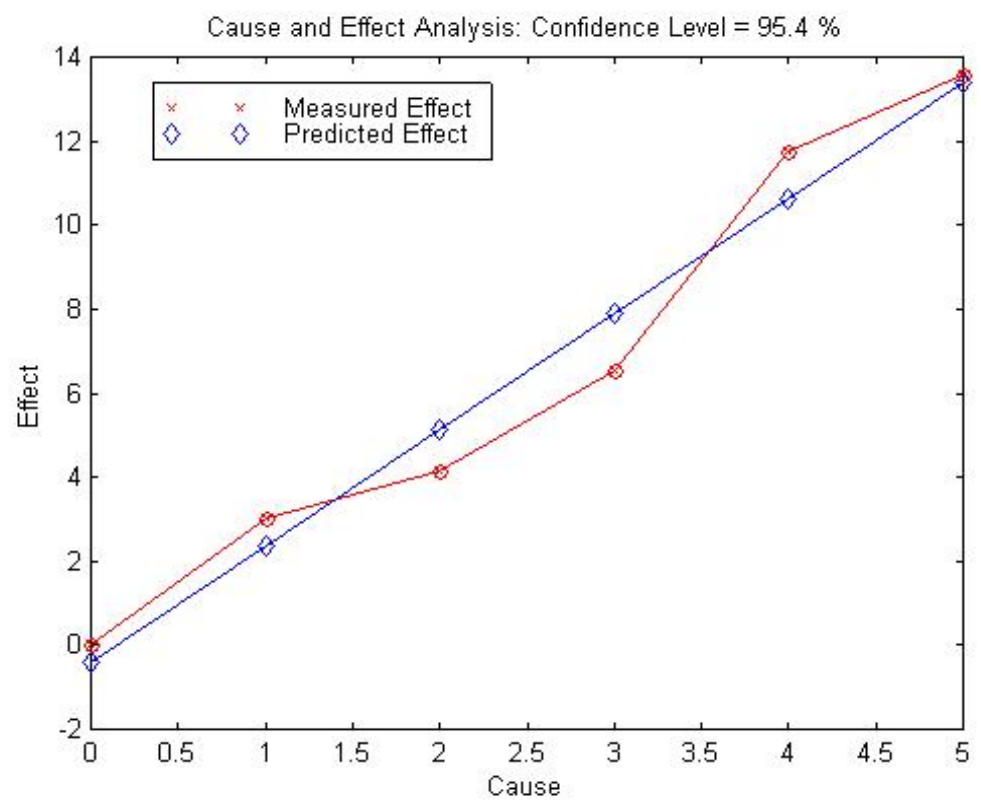

Figure 1. Illustrative example based upon a hypothetical computer-simulation.

multiples of a single initial input, in order to enable layperson evaluators to count on their five fingers, and both causes $C$ and effects $E$ were assumed to have the same physical dimensions (e.g. such as the integrated product of DC-current and voltage in Watt-seconds). In the sequel both of these assumptions are now relaxed.

\section{Analysis}

Let $N \geq 3$ denote the number of similarly prepared samples. Let the suffix $k$ denote any particular sample, $k=0,1,2, \ldots,(N-1)$, where the suffix $k=0$ is reserved for the case of a control blank. Let the non-negative quantities $\left\{C_{k}\right\}$, $k=0,1,2, \ldots,(N-1)$, denote the hypothesized causal inputs, where, as in Bass and Gleeson [2], each $C_{k}$ may be e.g. the result of continuous monitoring of the input electrical power [product of instantaneous voltage and current] and its numerical integration over the complete duration of the preparation of the $k^{\text {th }}$ sample to give the total amount $C_{k} \geq 0$ of electrical work done on the sample, or the total energy input. By definition, $C_{0}=0$. Similarly, let the non-negative quantities $\left\{E_{k}\right\}, k=$ $0,1,2, \ldots,(N-1)$, denote the measured effect outputs. Three possibilities for the output effects $\left\{E_{k}\right\}$ are:

(1) $E_{k}=$ amount of excess enthalpy;

(2) $E_{k}=$ amount of Helium-4;

(3) $E_{k}=$ amount of Helium-3. 
Now define the estimator of variance $\sigma^{2}$ by means of the following sum over all $k$ :

$$
\sigma^{2}=\frac{1}{(N-2)} \sum_{k=0}^{N-1}\left(E_{k}-d E-C_{k} \delta E\right)^{2}
$$

where $d E$ denotes mean effect-bias error, and where $\delta E$ denotes mean effectincrement factor. Next, again summing over all $k$, define

$$
\begin{gathered}
\alpha=\sum_{k=0}^{N-1} E_{k}, \\
\beta=\sum_{k=0}^{N-1} E_{k} C_{k}, \\
\gamma=\sum_{k=0}^{N-1} C_{k}, \\
\delta=\sum_{k=0}^{N-1}\left(C_{k}\right)^{2},
\end{gathered}
$$

and verify by setting to zero the gradient of $\sigma$ with respect to the column-vector $[d E, \delta E]^{T}$ that a necessary and sufficient condition for the sample standard deviation $\sigma$ to be minimized is that

$$
\begin{aligned}
d E & =\frac{(\delta \alpha-\gamma \beta)}{\Delta}, \\
\delta E & =\frac{(N \beta-\gamma \alpha)}{\Delta}, \\
\Delta & =N \delta-\gamma^{2} .
\end{aligned}
$$

Let

$$
\min C:=\min _{k>0}\left\{C_{k}\right\}
$$

and regard the analysis as a failure if the bias $d E$ is too large in the sense that

$$
|d E|>\frac{|\delta E| \min C}{2} .
$$

Finally, define the significance ratio 


$$
\rho=\frac{\sigma}{|\delta E| \min C}
$$

and note that if the errors have a Gaussian or "normal" distribution then the Confidence Level in the statistical significance of the result is at least $68.3 \%$ or $95.4 \%$ or $99.7 \%$ if $\rho<1$ or $\rho<1 / 2$ or $\rho<1 / 3$.

A MATLAB program available from the author implements the preceding analysis and presents the results in three different graphical forms, an example of the third of which is the following figure.

\section{Conclusions}

Instead of the usual eyeballing of a $(C, E)$ plot and assigning of a figure of merit regarding linearity in a subjective manner, the experimenter may now plan to evaluate a sequence of similar experiments in a completely objective manner by computing the significance ratio derived above.

The present protocol applies to systems near equilibrium (no output for no input, other than small erroneous effects attributable to disturbances or noise), and assumed to be sufficiently "smooth" as to be "locally" in the category of linear time-invariant (LTI) systems, e.g. not to become nonlinear (like a 'locally linear' amplifier which "saturates" for large inputs and so must be regarded as inherently nonlinear, globally) for the domain of inputs considered. Therefore the protocol

may be regarded as an objective procedure for testing the LTI hypothesis over the input domain considered.

\section{Acknowledgment}

We thank Ludwik Kowalski and Peter Hagelstein for advising us to clarify more explicitly the utility of the protocol derived herein.

\section{References}

1. R. W. Bass, "5 Frozen Needles CF Protocol," Journal of New Energy 6, pp. 30-32 (2001); and online at: http://www.lenr-canr.org/acrobat/BassRWfivefrozen.pdf.

2. R. W. Bass and W. S. Gleeson, "Theoretical and Experimental Results Regarding LENR/CF," Trans. of the American Nuclear Society 83 , pp. 355-56 (2000); and online at: http://www.padrak.com/ine/BASS_7.html. 